\title{
Similarity renormalization group for nucleon-nucleon interactions
}

\author{
S. K. Bogner, ${ }^{*}$ R. J. Furnstahl, ${ }^{\dagger}$ and R. J. Perry ${ }^{\ddagger}$ \\ Department of Physics, The Ohio State University, Columbus, Ohio 43210, USA
}

(Received 20 November 2006; published 13 June 2007)

\begin{abstract}
The similarity renormalization group (SRG) is based on unitary transformations that suppress off-diagonal matrix elements, forcing the Hamiltonian toward a band-diagonal form. A simple SRG transformation applied to nucleon-nucleon interactions leads to greatly improved convergence properties while preserving observables and provides a method to consistently evolve many-body potentials and other operators.
\end{abstract}

DOI: 10.1103/PhysRevC.75.061001

PACS number(s): 21.30.-x, 05.10.Cc, 13.75.Cs

Progress on the nuclear many-body problem has been hindered for decades because nucleon-nucleon $(N N)$ potentials that reproduce elastic-scattering phase shifts typically exhibit strong short-range repulsion as well as a strong tensor force. This leads to strongly correlated many-body wave functions and highly nonperturbative few- and many-body systems. But recent work shows how a cutoff on relative momentum can be imposed and evolved to lower values using renormalization group (RG) methods, thus eliminating the troublesome highmomentum modes [1,2]. The evolved $N N$ potentials are energy-independent and preserve two-nucleon observables for relative momenta up to the cutoff. Such potentials, known generically as $V_{\text {low } k}$, are more perturbative and generate much less correlated wave functions [2-7], vastly simplifying the many-body problem. However, a full RG evolution of essential few-body potentials has not yet been achieved.

An alternative path to decoupling high-momentum from low-momentum physics is the similarity renormalization group (SRG), which is based on unitary transformations that suppress off-diagonal matrix elements, driving the Hamiltonian toward a band-diagonal form [8-11]. The SRG potentials are automatically energy independent and have the feature that high-energy phase shifts (and other high-energy $N N$ observables), although typically highly model dependent, are preserved, unlike the case with $V_{\text {low } k}$ as usually implemented. Most important, the same transformations renormalize all operators, including many-body operators, and the class of transformations can be tailored for effectiveness in particular problems.

Here we make the first exploration of SRG for nucleonnucleon interactions, using a particularly simple choice of SRG transformation, which nevertheless works exceedingly well. We find the same benefits of $V_{\text {low } k}$ : more perturbative interactions and lessened correlations, with improved convergence in few- and many-body calculations. The success of the SRG combined with advances in chiral effective field theory (EFT) $[12,13]$ opens the door to the consistent construction and RG evolution of many-body potentials and other operators.

The similarity RG approach was developed independently by Glazek and Wilson [8] and by Wegner [9]. We follow

\footnotetext{
*bogner@mps.ohio-state.edu

†furnstahl.1@osu.edu

†perry.6@osu.edu
}

Wegner's formulation in terms of a flow equation for the Hamiltonian. The initial Hamiltonian in the center-of-mass $H=T_{\text {rel }}+V$, where $T_{\text {rel }}$ is the relative kinetic energy, is transformed by the unitary operator $U(s)$ according to

$$
H_{s}=U(s) H U^{\dagger}(s) \equiv T_{\text {rel }}+V_{s},
$$

where $s$ is the flow parameter. This also defines the evolved potential $V_{s}$, with $T_{\text {rel }}$ taken to be independent of $s$. Then $H_{s}$ evolves according to

$$
\frac{d H_{s}}{d s}=\left[\eta(s), H_{s}\right]
$$

with

$$
\eta(s)=\frac{d U(s)}{d s} U^{\dagger}(s)=-\eta^{\dagger}(s) .
$$

Choosing $\eta(s)$ specifies the transformation. Here we make perhaps the simplest choice [10],

$$
\eta(s)=\left[T_{\mathrm{rel}}, H_{s}\right]
$$

which gives the flow equation,

$$
\frac{d H_{s}}{d s}=\left[\left[T_{\mathrm{rel}}, H_{s}\right], H_{s}\right] .
$$

Other choices will be studied elsewhere [14].

For any given partial wave in the space of relative momentum $N N$ states, Eq. (5) means that the potential in momentum space evolves as (with normalization so that $1=\frac{2}{\pi} \int_{0}^{\infty}|q\rangle q^{2} d q\langle q|$ and in units where $\left.\hbar^{2} / M=1\right)$,

$$
\begin{aligned}
\frac{d V_{s}\left(k, k^{\prime}\right)}{d s}= & -\left(k^{2}-k^{\prime 2}\right)^{2} V_{s}\left(k, k^{\prime}\right) \\
& +\frac{2}{\pi} \int_{0}^{\infty} q^{2} d q\left(k^{2}+k^{\prime 2}-2 q^{2}\right) \\
& \times V_{s}(k, q) V_{s}\left(q, k^{\prime}\right) .
\end{aligned}
$$

(The additional matrix structure of $V_{s}$ in coupled channels such as ${ }^{3} \mathrm{~S}_{1}-{ }^{3} \mathrm{D}_{1}$ is implicit.) For matrix elements far from the diagonal, the first term on the right side of Eq. (6) evidently dominates and exponentially suppresses these elements as $s$ increases. The parameter $\lambda \equiv s^{-1 / 4}$ provides a measure of the spread of off-diagonal strength. Although further analytic analysis is possible, we instead turn to a numerical demonstration that the flow toward the diagonal is a general 

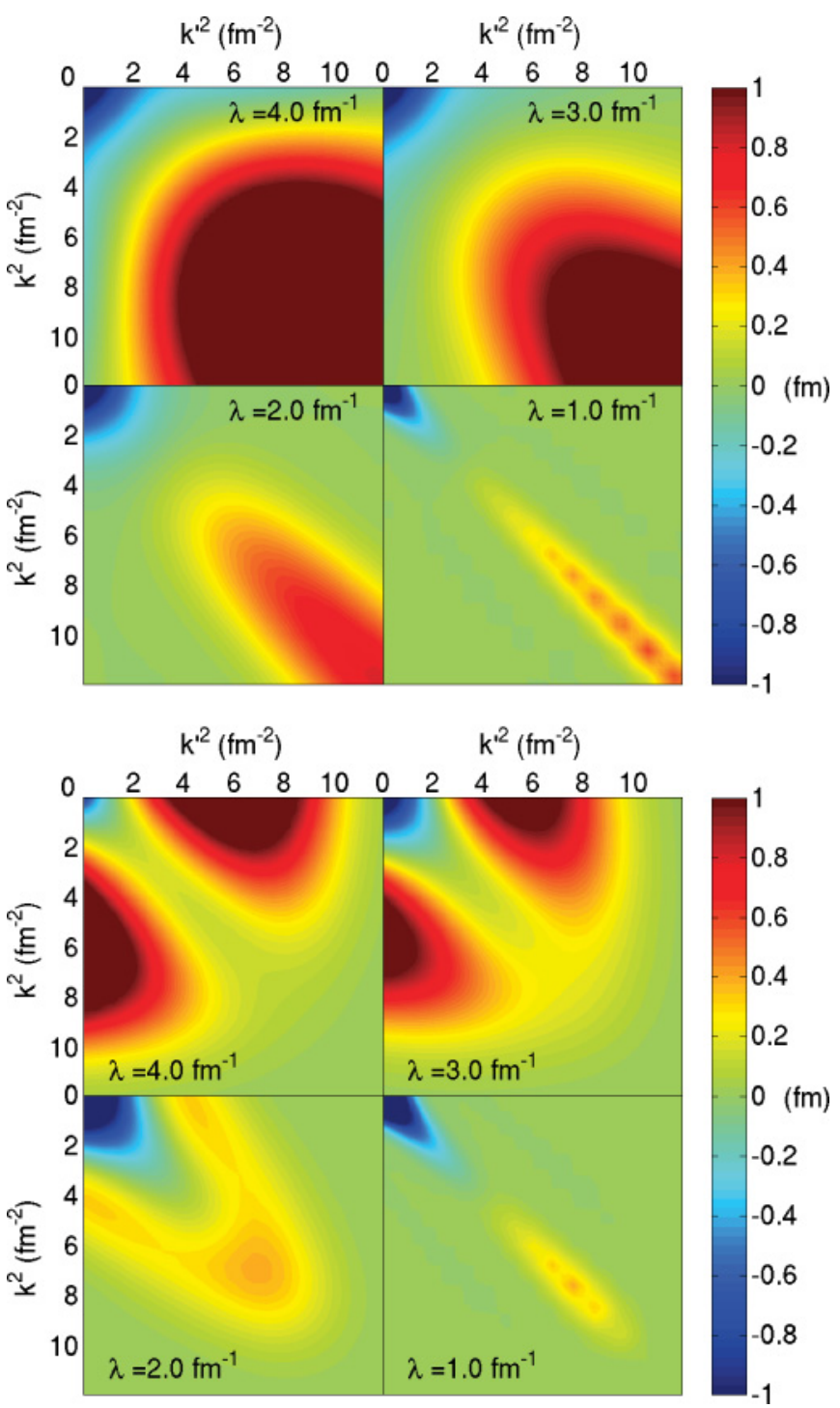

FIG. 1. (Color online) Contour plots of $V_{s}\left(k, k^{\prime}\right)$ illustrating the evolution with $\lambda \equiv s^{-1 / 4}$ for ${ }^{1} \mathrm{~S}_{0}$ (left) and ${ }^{3} \mathrm{~S}_{1}$ (right). The initial potential on the left is a chiral $\mathrm{N}^{3} \mathrm{LO}$ potential with a $600-\mathrm{MeV}$ cutoff [12] and on the right is an $\mathrm{N}^{3} \mathrm{LO}$ potential with a $550-\mathrm{MeV}$ cutoff on the Lippmann-Schwinger equation and a $600-\mathrm{MeV}$ cutoff on a regularized spectral representation of two-pion exchange [13]. result. By discretizing the relative momentum space on a grid of Gaussian integration points, we obtain a simple (but nonlinear) system of first-order coupled differential equations, with the boundary condition that $V_{s}\left(k, k^{\prime}\right)$ at the initial $s$ (or $\lambda$ ) is equal to the initial potential.

The evolution of the Hamiltonian according to Eq. (6) as $s$ increases (or $\lambda$ decreases) is illustrated in Fig. 1, using two initial chiral EFT potentials [12,13]. On the left in Fig. 1 is ${ }^{1} \mathrm{~S}_{0}$ starting from the harder $(600-\mathrm{MeV}$ cutoff) potential from Ref. [12], which has significant strength near the highmomentum diagonal, and on the right is the $S$-wave part of the ${ }^{3} \mathrm{~S}_{1}-{ }^{3} \mathrm{D}_{1}$ coupled channel starting from one of the potentials from Ref. [13], which has more far off-diagonal strength initially and comparatively weaker higher-momentum strength on the diagonal. The initial momentum-space potential differs significantly among interactions that are phase equivalent up to the $N N$ inelastic threshold, but these examples show characteristic features of the evolution in $\lambda$. In particular, we see a systematic suppression of off-diagonal strength, as anticipated, with the width of the diagonal scaling as $\lambda^{2}$. The same behavior is observed when evolving from conventional high-precision $N N$ potentials, such as Argonne $v_{18}$, or other (softer) chiral potentials $[14,15]$.

Because the SRG transformation is unitary, observables are unchanged at all energies, up to numerical errors. This is shown by Fig. 2, in which phase shifts for the two chiral EFT potentials are plotted, including the values at high energies where they are not constrained by data (above $E_{\mathrm{lab}}=300 \mathrm{MeV}$ ). For a given potential, there is no visible variation with $\lambda$. Similarly, the binding energy and asymptotic normalizations for the deuteron are independent of $\lambda[15]$.

As $\lambda$ is lowered, different initial potentials flow to similar forms at low momentum while remaining distinct at higher momentum. The low-momentum parts also become similar to $V_{\text {low } k}$ potentials. These observations are illustrated in Fig. 3 for two particular slices of the potentials from Fig. 1. They will be explored in much greater detail in Ref. [14].

We can quantify the perturbativeness of the potential as we evolve to lower $\lambda$ by using the eigenvalue analysis introduced long ago by Weinberg [16] and recently applied in an analysis of $V_{\text {low } k}$ potentials [6]. Consider the operator Born series for
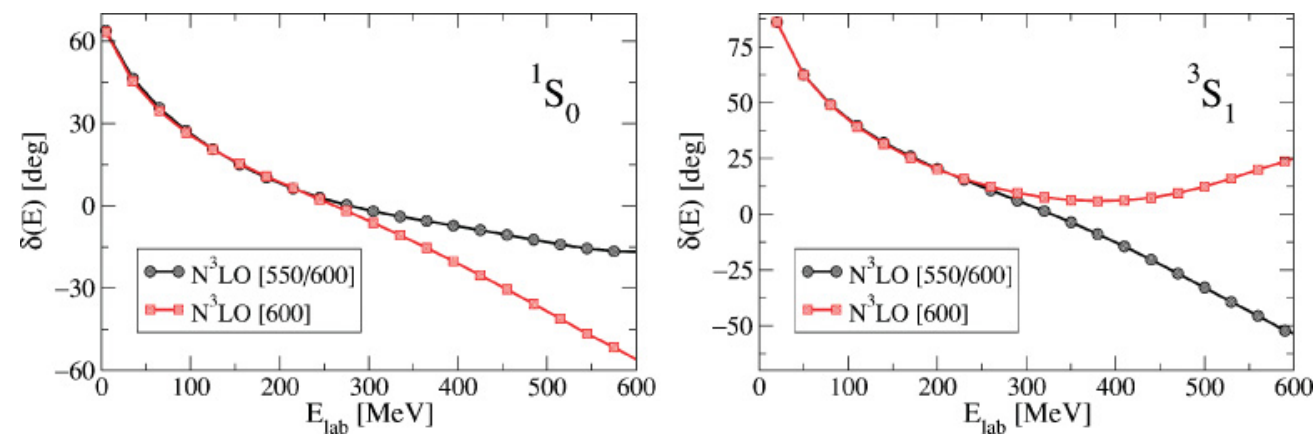

FIG. 2. (Color online) $S$-wave phase shifts from the two chiral EFT $N^{3}$ LO potentials from Fig. 1. For each initial potential, the phase shifts for different $\lambda$ agree to within the widths of the lines at all energies shown. 


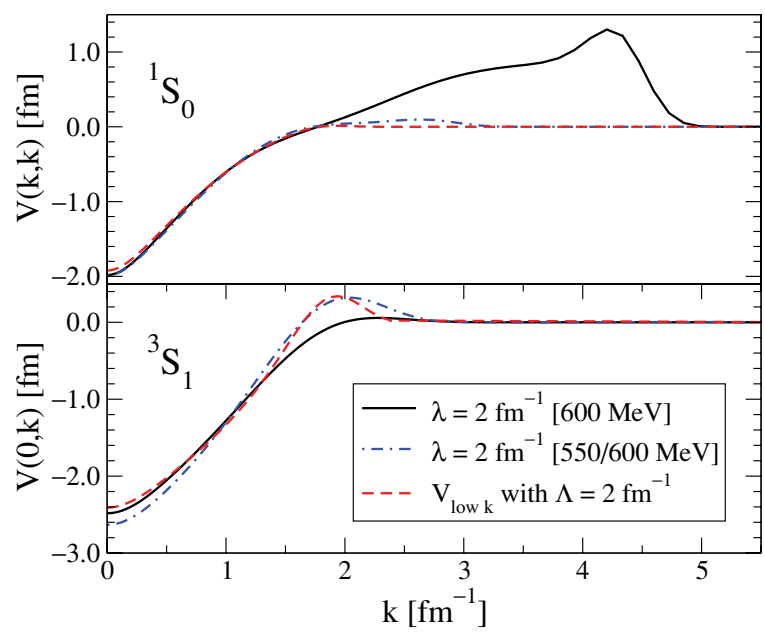

FIG. 3. (Color online) Matrix elements of the evolved SRG potentials at $\lambda=2 \mathrm{fm}^{-1}$ for ${ }^{1} \mathrm{~S}_{0}$ (top, diagonal elements) and ${ }^{3} \mathrm{~S}_{1}$ (bottom, off-diagonal elements) for the same initial potentials as in Fig. 1. Also shown is the $V_{\text {low } k}$ potential with a smooth (exponential) regulator for momentum cutoff $\Lambda=2 \mathrm{fm}^{-1}$, evolved from the two potentials (600 $\mathrm{MeV}$ above and 550/600 $\mathrm{MeV}$ below).

the $T$-matrix at energy $E$ (for simplicity we assume $E \leqslant 0$ ):

$$
T(E)=V_{s}+V_{s} \frac{1}{E-T_{\text {rel }}} V_{s}+\ldots
$$

By finding the eigenvalues and eigenvectors of

$$
\frac{1}{E-T_{\text {rel }}} V_{s}\left|\Gamma_{\nu}\right\rangle=\eta_{\nu}(E)\left|\Gamma_{\nu}\right\rangle,
$$

and then acting with $T(E)$ on the eigenvectors,

$$
T(E)\left|\Gamma_{v}\right\rangle=V_{s}\left|\Gamma_{v}\right\rangle\left(1+\eta_{v}+\eta_{v}^{2}+\ldots\right),
$$

it follows that nonperturbative behavior at energy $E$ is signaled by one or more eigenvalues with $\left|\eta_{\nu}(E)\right| \geqslant 1$ [16]. (See Ref. [6] for a more detailed discussion in the context of evolving $V_{\text {low } k}$ potentials.)

It suffices for our purposes to consider a single energy (e.g., $E=0$ ), and to consider only the negative eigenvalues, which are associated with the short-range repulsion. Weinberg eigenvalues at zero energy are shown as a function of $\lambda$ in Fig. 4 for the ${ }^{1} S_{0}$ channel and the ${ }^{3} S_{1}{ }^{3} D_{1}$ coupled channel. In both channels, the large negative eigenvalues at large $\lambda$ reflect the repulsive core of the initial potentials. They rapidly evolve to small values as $\lambda$ decreases to $2 \mathrm{fm}^{-1}$ and below, as also observed with the corresponding $V_{\text {low } k}$ evolution [6]. However, the intermediate increase for the subleading eigenvalues in ${ }^{1} \mathrm{~S}_{0}$ is a new feature of the SRG that merits further study.

The more perturbative potentials at lower $\lambda$ induce weaker short-range correlations in few- and many-body wave functions, which in turn leads to greatly improved convergence in variational calculations. This is illustrated via calculations of the binding energy of the deuteron and triton by diagonalization in a harmonic oscillator basis, as shown in Figs. 5 and 6. For a fixed basis size, a more accurate estimate is obtained with smaller $\lambda$ or, conversely, at fixed $\lambda$ the convergence with basis size becomes more rapid. The improvement in convergence is

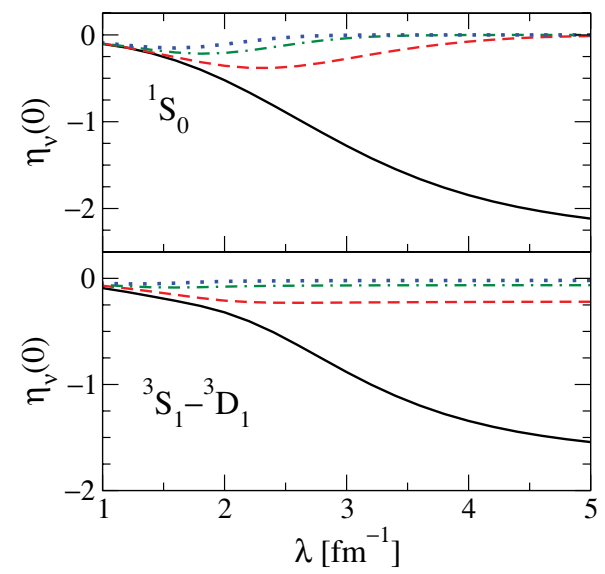

FIG. 4. (Color online) The largest repulsive Weinberg eigenvalues as a function of $\lambda$ in the ${ }^{1} S_{0}$ channel and the ${ }^{3} S_{1}-{ }^{3} D_{1}$ coupled channel for the same initial potentials as in Fig. 1.

similar to that found with smoothly regulated $V_{\text {low } k}$ potentials evolved from chiral $\mathrm{N}^{3} \mathrm{LO}$ potentials [7]. At finite density, analogous effects led to perturbative behavior in nuclear matter for $V_{\text {low } k}$ potentials [5]. Results for the $G$-matrix support a similar conclusion for the SRG potentials (see the Web site in Ref. [14] for pictures).

In Fig. 6 , the calculations for different $\lambda$ converge to different values for the binding energy of the triton. This reflects the contributions of the omitted (and evolving) threebody interaction. The evolution with $\lambda$ of the binding energy with $N N$ interactions only, which is also the evolution of the net three-body contribution, follows a similar pattern to that seen with $V_{\text {low } k}[4,7]$ : a slow decrease as $\lambda$ decreases, reaching a minimum for $\lambda$ between 1.5 and $2 \mathrm{fm}^{-1}$, and then a rapid increase.

The consistent RG evolution of few-body interactions is an important unsolved problem for low-momentum potentials. In $V_{\text {low } k}$ calculations to date, an approximate evolution is made by fitting the leading chiral EFT three-body force at

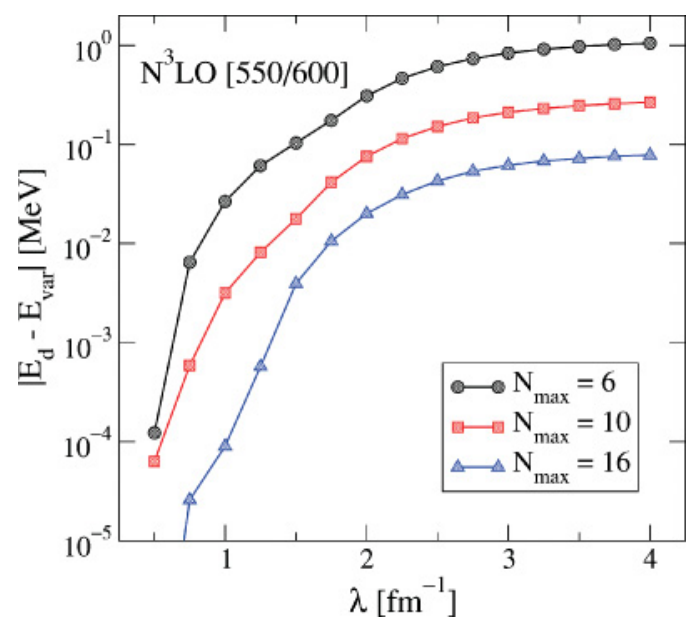

FIG. 5. (Color online) The absolute error vs. $\lambda$ of the predicted deuteron binding energy from a variational calculation in a fixedsize basis of harmonic oscillators ( $N_{\max } \hbar \omega$ excitations). The initial potential is from Ref. [13]. 


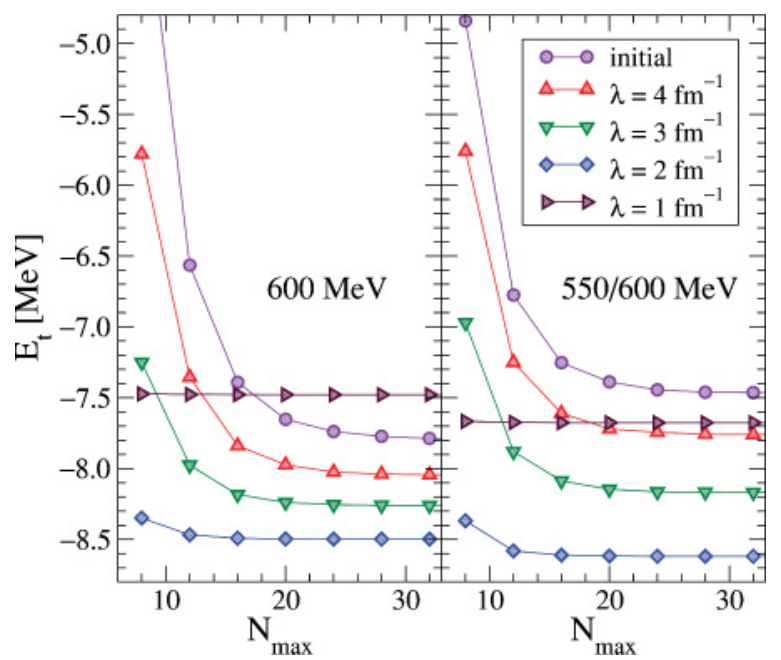

FIG. 6. (Color online) The variational binding energy for selected $\lambda$ of the triton with two-nucleon interactions only, as a function of the size of the harmonic oscillator space ( $N_{\max } \hbar \omega$ excitations), for the same initial potentials as in Fig. 1.

each cutoff while evolving the two-body interaction exactly [4]. Generalizing the RG evolution to include the three-body interaction is, at least, technically challenging. This is because the machinery used to construct $V_{\text {low } k}$ requires the solution of the full three-nucleon problem (i.e., bound state wave functions plus all scattering wave functions in all breakup channels) to consistently evolve the three-nucleon interactions.

In contrast, the SRG evolution follows by directly applying Eq. (5) in the three-particle space. To show the basic idea, we adopt a notation in which $V_{12}$ means the two-body interaction between particles 1 and 2, whereas $V_{123}$ is the irreducible threebody potential. In standard treatments of the nuclear threebody problem (for example, Ref. [17]), the relative kinetic energy for equal mass particles with mass $m$ is decomposed as (with the total momentum $\mathbf{K}=\sum_{i} \mathbf{k}_{i}=0$ ):

$$
T_{\text {rel }}=\sum_{i} \frac{\mathbf{k}_{i}^{2}}{2 m}=\frac{\mathbf{p}_{l}^{2}}{m}+\frac{3 \mathbf{q}_{l}^{2}}{4 m}, \quad l=1,2,3,
$$

where $l$ denotes which set of Jacobi coordinates are being used to describe the relative motion (i.e., $l=1$ means $\mathbf{p}_{1}=$ $\frac{1}{2}\left(\mathbf{k}_{2}-\mathbf{k}_{3}\right)$ and $\mathbf{q}_{1}=\frac{2}{3}\left[\mathbf{k}_{1}-\frac{1}{2}\left(\mathbf{k}_{2}+\mathbf{k}_{3}\right)\right]$, and so on $)$. In the notation here,

$$
\frac{\mathbf{p}_{1}^{2}}{m} \leftrightarrow T_{23} \quad \text { and } \quad \frac{3 \mathbf{q}_{1}^{2}}{4 m} \leftrightarrow T_{1}
$$

and similarly for $l=2$ and $l=3$.

Now we start with the Hamiltonian including up to three-body interactions (keeping in mind that higher-body interactions will be induced as we evolve in $s$ but will not contribute to three-body systems):

$$
H_{s}=T_{\text {rel }}+V_{12}+V_{13}+V_{23}+V_{123} \equiv T_{\text {rel }}+V_{s} .
$$

(Note: all of the potentials depend implicitly on $s$.) The relative kinetic energy operator $T_{\text {rel }}$ can be decomposed in three ways:

$$
T_{\text {rel }}=T_{12}+T_{3}=T_{13}+T_{2}=T_{23}+T_{1},
$$

and $T_{i}$ commutes with $V_{j k}$,

$$
\left[T_{3}, V_{12}\right]=\left[T_{2}, V_{13}\right]=\left[T_{1}, V_{23}\right]=0,
$$

so the commutators of $T_{\text {rel }}$ with the two-body potentials become

$$
\left[T_{\text {rel }}, V_{12}\right]=\left[T_{12}, V_{12}\right],
$$

and similarly for $V_{13}$ and $V_{23}$.

Because we define $T_{\text {rel }}$ to be independent of $s$, the SRG equation (5) for the three-body Hamiltonian $H_{s}$ simplifies to

$$
\frac{d V_{s}}{d s}=\frac{d V_{12}}{d s}+\frac{d V_{13}}{d s}+\frac{d V_{23}}{d s}+\frac{d V_{123}}{d s}=\left[\left[T_{\mathrm{rel}}, V_{s}\right], H_{s}\right],
$$

with $V_{s}$ from Eq. (12). The corresponding equations for each of the two-body potentials (which are completely determined by their evolved matrix elements in the two-body systems, e.g., on a Gaussian momentum grid) are

$$
\frac{d V_{12}}{d s}=\left[\left[T_{12}, V_{12}\right],\left(T_{12}+V_{12}\right)\right],
$$

and similarly for $V_{13}$ and $V_{23}$. After expanding Eq. (16) using Eq. (12) and the different decompositions of $T_{\text {rel }}$, it is straightforward to show using the equations for the two-body potentials that the derivatives of two-body potentials on the left side cancel precisely with terms on the right side, leaving

$$
\begin{aligned}
\frac{d V_{123}}{d s}= & {\left[\left[T_{12}, V_{12}\right],\left(T_{3}+V_{13}+V_{23}+V_{123}\right)\right] } \\
& +\left[\left[T_{13}, V_{13}\right],\left(T_{2}+V_{12}+V_{23}+V_{123}\right)\right] \\
& +\left[\left[T_{23}, V_{23}\right],\left(T_{1}+V_{12}+V_{13}+V_{123}\right)\right] \\
& +\left[\left[T_{\text {rel }}, V_{123}\right], H_{s}\right] .
\end{aligned}
$$

The importance of these cancellations is that they eliminate the "dangerous" delta functions, which make setting up the integral equations for the three-body system problematic [17]. We emphasize that the $s$ dependence of the two-body interactions on the right side of Eq. (18) is completely specified by solving the two-body problem in Eq. (6). This is in contrast to RG methods that run a cutoff on the total energy of the basis states (e.g., Lee-Suzuki and Bloch-Horowitz techniques). Such methods generate "multivalued" two-body interactions, in the sense that the RG evolution of two-body operators in $A>2$ systems depends nonlocally on the excitation energies of the unlinked spectator particles $[18,19]$.

Further simplications of Eq. (18) follow from symmetrization and applying the Jacobi identity, but this form is sufficient to make clear that there are no disconnected pieces. The problem is thus reduced to the technical implementation of a momentum-space decomposition analogous to Eq. (6). Note in particular that the evolution of $V_{123}$ is carried out without ever having to solve a bound-state or scattering problem. 
We have verified that this formalism does generate threebody interactions that leave eigenvalues invariant for simple model Hamiltonians, such as a two-level system of bosons. Work is in progress on proof-of-principle tests using onedimensional many-body systems (to avoid angular momentum complications) and the three-dimensional nuclear problem will be tackled soon.

In summary, the SRG applied to nucleon-nucleon potentials works as advertised even for a simple choice of transformation, driving the Hamiltonian (in momentum space) toward the diagonal, making it more perturbative and more convergent in few-body calculations. There is much to explore, such as the nature of the decoupling of high- and low-energy physics implied by Fig. 1 (see Ref. [15] for demonstrations of decoupling and the evolution of an operator in the two-body space) and whether other choices of $\eta(s)$ instead of Eq. (4) could be more effective in making the Hamiltonian diagonal. For example, the replacement $T_{\text {rel }} \rightarrow H_{d}$, where $H_{d}$ is the diagonal part of the Hamiltonian, or some function of $T_{\text {rel }}$ are easily implemented. Most important is the consistent evolution of nuclear three-body operators [14].

We thank A. Schwenk for useful comments. This work was supported in part by the National Science Foundation under grant PHY-0354916.
[1] E. Epelbaum, W. Glöckle, A. Krüger, and U. G. Meißner, Nucl. Phys. A645, 413 (1999).

[2] S. K. Bogner, T. T. S. Kuo, and A. Schwenk, Phys. Rep. 386, 1 (2003).

[3] S. K. Bogner, A. Schwenk, T. T. S. Kuo, and G. E. Brown, nucl-th/0111042.

[4] A. Nogga, S. K. Bogner, and A. Schwenk, Phys. Rev. C 70, 061002(R) (2004).

[5] S. K. Bogner, A. Schwenk, R. J. Furnstahl, and A. Nogga, Nucl. Phys. A763, 59 (2005).

[6] S. K. Bogner, R. J. Furnstahl, S. Ramanan, and A. Schwenk, Nucl. Phys. A773, 203 (2006).

[7] S. K. Bogner, R. J. Furnstahl, S. Ramanan, and A. Schwenk, Nucl. Phys. A784, 79 (2007).

[8] S. D. Glazek and K. G. Wilson, Phys. Rev. D 48, 5863 (1993); 49, 4214 (1994).

[9] F. Wegner, Ann. Phys. (Leipzig) 3, 77 (1994).

[10] S. Szpigel and R. J. Perry, in Quantum Field Theory, A 20th Century Profile, edited by A. N. Mitra (Hindustan, New Delhi, 2000).

[11] An alternative non-RG use of unitary transformations to reduce correlations in many-body wave functions is described in R. Roth, H. Hergert, P. Papakonstantinou, T. Neff, and H. Feldmeier, Phys. Rev. C 72, 034002 (2005), and references therein. The relationship of this approach to the SRG is described in H. Hergert and R. Roth, Phys. Rev. C 75, 051001(R) (2007).

[12] D. R. Entem and R. Machleidt, Phys. Rev. C 68, 041001(R) (2003).

[13] E. Epelbaum, W. Glöckle, and U. G. Meißner, Nucl. Phys. A747, $362(2005)$.

[14] S. K. Bogner, R. J. Furnstahl, and R. J. Perry, in preparation. See also http://www.physics.ohio-state.edu/ ntg/srg/ for documentary examples of the SRG applied to $N N$ potentials.

[15] S. K. Bogner, R. J. Furnstahl, R. J. Perry, and A. Schwenk, Phys. Lett. B649, 488 (2007).

[16] S. Weinberg, Phys. Rev. 131, 440 (1963).

[17] W. Glöckle, The Quantum Mechanical Few-Body Problem (Springer-Verlag, Berlin, 1983).

[18] P. Navratil and B. R. Barrett, Phys. Rev. C 54, 2986 (1996).

[19] T. C. Luu, S. K. Bogner, W. C. Haxton, and P. Navratil, Phys. Rev. C 70, 014316 (2004).

[20] H. Hergert and R. Roth, Phys. Rev. C 75, 051001(R) (2007). 